Pacific Journal of Mathematics

GENERALIZATION OF LENTIN'S THEORY OF PRINCIPAL DDITION 


\title{
GENERALIZATION OF LENTIN'S THEORY OF PRINCIPAL SOLUTIONS OF WORD EQUATIONS IN FREE SEMIGROUPS TO FREE PRODUCT OF COPIES OF POSITIVE REALS UNDER ADDITION
}

\author{
Mohan S. PUTCha
}

\begin{abstract}
Word equations in free semigroups have been studied by many authors. However, word equations in geometrically arising semigroups lead to word equations in free product of copies of positive reals under addition (see "Word equations in some geometric semigroups" by Putcha). In this paper we generalize Lentin's theory of word equations in free semigroups to free product of copies of positive reals under addition.
\end{abstract}

1. Preliminaries. Throughout this paper $Z_{0}, Z^{+}, Z, Q_{0}, Q^{+}, Q$, $\boldsymbol{R}_{0}, \boldsymbol{R}^{+}, \boldsymbol{R}$ will denote the sets of nonnegative integers, positive integers, integers, nonnegative rational numbers, positive rational numbers, rational numbers, nonnegative real numbers, positive real numbers and reals, respectively. If $S$ is a semigroup without an identity element, then $S^{1}=S \cup\{1\}$ with obvious multiplication. If $a, b \in S$, then $\left.a\right|_{f} b$ ( $a$ is a final segment of $b$ ) if $b=x a$ for some $x \in S^{1}$.

If $\Gamma$ is a nonempty set, then let $\mathscr{F}=\mathscr{F}(\Gamma)$ denote the free semigroup on $\Gamma$. If $w \in \mathscr{F}$, then let $l(w)=$ length of $w$. Let $\mathscr{F}_{\boldsymbol{R}}=\mathscr{F}_{\boldsymbol{R}}(\Gamma)$ denote the set of all nonempty finite sequences (also called words) of the type $w=A_{1}^{\alpha_{1}} \cdots A_{n}^{\alpha_{n}}$ where $n \in Z^{+}, \alpha_{1}, \cdots, \alpha_{n} \in$ $\boldsymbol{R}^{+}, A_{1}, \cdots, A_{n} \in \Gamma$ and $A_{i} \neq A_{i+1}$ for $i, i+1 \in\{1, \cdots, n\}$. We define $e(w)=n$ and $l(w)=\alpha_{1}+\cdots+\alpha_{n}$. Let $w_{1}, w_{2} \in \mathscr{F}_{R}$. Suppose $w_{1}=$ $A_{1}^{\alpha_{1}} \cdots A_{n}^{\alpha_{n}}, w_{2}=B_{1}^{\beta_{1}} \cdots B_{m}^{\beta_{m}}$. Then we define

$$
w_{1} w_{2}=\left(\begin{array}{lll}
A_{1}^{\alpha_{1}} \cdots A_{n}^{\alpha_{n}+\beta_{1}} B_{2}^{\beta_{2}} \cdots B_{m}^{\beta_{m}} & \text { if } & A_{n}=B_{1} \\
A_{1}^{\alpha_{1}} \cdots A_{n}^{\alpha_{n}} B_{1}^{\beta_{1}} \cdots B_{m}^{\beta_{m}} & \text { if } & A_{n} \neq B_{1} .
\end{array}\right.
$$

Now of course expressions of the type $w=A_{1}^{\alpha_{1}} \cdots A_{n}^{\alpha_{n}}\left(\alpha_{1}, \cdots, \alpha_{n} \in\right.$ $\left.\boldsymbol{R}^{+} ; A_{1}, \cdots, A_{n} \in \Gamma\right)$ make sense even when $A_{i}=A_{i+1}$ for some $i$, $i+1 \in\{1, \cdots, n\}$. But note that if $n=e(w)$, then $A_{i} \neq A_{i+1}$ for any $i, i+1 \in\{1, \cdots, n\}$. In such a case we call $A_{1}^{\alpha_{1}} \cdots A_{n}^{\alpha_{n}}$, the standard form of $w . \mathscr{F}_{\mathbf{R}}(\Gamma)$ is a semigroup and is just the free product of $|\Gamma|$ copies of $\boldsymbol{R}^{+}$under addition (see for example [3; p. 411]). If $w \in \mathscr{F}_{\boldsymbol{R}}{ }^{1}$, then we let $w^{0}=1$. Let $\bar{\Gamma}=\left\{A^{\alpha} \mid A \in \Gamma, \alpha \in \boldsymbol{R}^{+}\right\}$. If $u, v \in \bar{\Gamma}$, define $u \sim v$ if $u=A^{\alpha}, v=A^{\beta}$ for some $\alpha, \beta \in R^{+}, A \in \Gamma$. Let $w \in \mathscr{F}_{R}$, 
$w=A_{1}^{\alpha_{1}} \cdots A_{n}^{\alpha_{n}}$ in standard form. Then we say that $w$ starts with $A_{1}$ and ends with $A_{n}$. Let $A \in \Gamma$. Then $A$ appears in $w$ if $A=A_{i}$ for some $i \in\{1, \cdots, n\} . A$ appears integrally in $w$ if for each $i \in\{1, \cdots, n\}, A_{i}=A$ implies $\alpha_{i} \in Z^{+}$. Otherwise $A$ appears nonintegrally in $w$. Note that if $A$ does not appear in $w$, then $A$ appears integrally in $w$. A appears rationally in $w$ if for each $i \in\{1, \cdots, n\}$, $A_{i}=A$ implies $\alpha_{i} \in Q^{+}$. Let $\mathscr{F}_{Q}(\Gamma)=\left\{w \mid w \in \mathscr{F}_{\boldsymbol{R}}(\Gamma), A\right.$ appears rationally in $w$ for each $A \in \Gamma\}$. If $\Lambda \subseteq \Gamma$, then let $\mathscr{F}_{R}(\Gamma \mid \Lambda)=\left\{w \mid w \in \mathscr{F}_{R}(\Gamma)\right.$, $A$ appears integrally in $w$ for each $A \in \Lambda\}$. So $\mathscr{F}_{\boldsymbol{R}}(\Gamma \mid \Gamma)=\mathscr{F}(\Gamma)$ and $\mathscr{F}_{R}(\Gamma \mid \varnothing)=\mathscr{F}_{R}(\Gamma)$. Also let $\mathscr{F}_{Q}(\Gamma \mid \Lambda)=\mathscr{F}_{R}(\Gamma \mid \Lambda) \cap \mathscr{F}_{Q}(\Gamma)$.

Let $\Lambda_{1} \cong \Gamma_{1}, \Lambda_{2} \subseteq \Gamma_{2}, \phi: \Gamma_{1} \rightarrow \mathscr{F}_{\mathbf{R}}\left(\Gamma_{2} \mid \Lambda_{2}\right)$ such that $\phi\left(\Gamma_{1} \backslash \Lambda_{1}\right) \subseteq \overline{\Gamma_{2} \backslash \Lambda_{2}}$. Then we can extend $\phi$ to a homomorphism $\hat{\phi}: \mathscr{F}_{\boldsymbol{R}}\left(\Gamma_{1} \mid \Lambda_{1}\right) \rightarrow \mathscr{F}_{\boldsymbol{R}}\left(\Gamma_{2} \mid \Lambda_{2}\right)$ by letting $\hat{\phi}\left(A^{\alpha}\right)=[\phi(A)]^{\alpha}$ for $A \in \Gamma_{1} \backslash \Lambda_{1}, \alpha \in \boldsymbol{R}^{+}$. We call $\hat{\phi}$ the natural extension of $\phi$. Let $\psi: \mathscr{F}_{R}\left(\Gamma_{1} \backslash \Lambda_{1}\right) \rightarrow \mathscr{F}_{R}\left(\Gamma_{2} \backslash \Lambda_{2}\right)$ be a homomorphism. Let $A \in \Gamma_{1} \backslash \Lambda_{1}$. Then $A$ and hence $\psi(A)$ has $n$th roots for all $n \in Z^{+}$. It follows that $\psi(A) \in \overline{\Gamma_{2} \backslash \Lambda_{2}}$. We say that is is a natural homomorphism if $\psi\left(A^{\alpha}\right)=\psi(A)^{\alpha}$ for all $A \in \Gamma_{1} \backslash \Lambda_{1}, \alpha \in \boldsymbol{R}^{+}$. In such a case $\psi=\hat{\phi}$ where $\phi$ is the restriction of $\psi$ to $\Gamma_{1}$. In this paper we only consider natural homomorphisms.

DeFinition 1.1. By a word equation in variables $X_{1}, \cdots, X_{n}$ we mean $\left\{w_{1}, w_{2}\right\}$ where $w_{1}=w_{1}\left(X_{1}, \cdots, X_{n}\right), w_{2}=w_{2}\left(X_{1}, \cdots, X_{n}\right) \in$ $\mathscr{F}\left(X_{1}, \cdots, X_{n}\right)^{1}$. It is not necessary that each $X_{i}$ appears in $w_{1} w_{2}$. Let $S$ be a semigroup and $a_{1}, \cdots, a_{n} \in S$. Then $\left(a_{1}, \cdots, a_{n}\right)$ is a solution of $\left\{w_{1}, w_{2}\right\}$ if $w_{1}\left(a_{1}, \cdots, a_{n}\right)=w_{2}\left(a_{1}, \cdots, a_{n}\right)$.

Detailed study of word equations leads to the concept of a generalized word equation defined below. This concept is similar but not identical to the concept of a constrained word equation defined in [5].

Definition. By a generalized word equation in variables $X_{1}, \cdots, X_{n}$ we mean $\mathscr{V}=\left\{w_{1}, w_{2} ; T_{1}, \cdots, T_{s}\right\}$ where $w_{1}=w_{1}\left(X_{1}, \cdots, X_{n}\right)$, $w_{2}=w_{2}\left(X_{1}, \cdots, X_{n}\right) \in \mathscr{F}\left(X_{1}, \cdots, X_{n}\right)^{1}$ and $T_{1}, \cdots, T_{s}$ are pairwise disjoint nonempty subsets of $\left\{X_{1}, \cdots, X_{n}\right\} . \quad(s=0$ means that $\mathscr{P}$ is the word equation $\left\{w_{1}, w_{2}\right\}$.) Let $X \in\left\{X_{1}, \cdots, X_{n}\right\}$. Then $X$ is a constrained variable if $X \in T_{i}$ for some $i$. Otherwise $X$ is a free variable of $\mathscr{W}$.

Let $u_{1}, \cdots, u_{n} \in \mathscr{F}_{\mathbf{R}}(\Omega)$. Then $\mu=\left(u_{1}, \cdots, u_{n}\right)$, is a solution of प्f if (i) $w_{1}\left(u_{1}, \cdots, u_{n}\right)=w_{2}\left(u_{1}, \cdots, u_{n}\right)$, (ii) $X_{i} \in T_{j}$ implies $e\left(u_{i}\right)=1$, and (iii) $X_{i}, X_{k} \in T_{j}$ implies $u_{i} \sim u_{k}$. Let $\Omega_{1}=\{A \mid A \in \Omega, A$ appears in $\left.u_{1} \cdots u_{n}\right\}$ and $\Omega_{2}=\left\{A \mid A \in \Omega, A\right.$ appears integrally in each $u_{i}$, $i=1, \cdots, n\}$. We define the symbol $\Omega_{1} \mid \Omega_{2}$ to be the alphabet of $\mu$. If $\Omega_{1}=\Omega_{2}$ we also write $\Omega_{1}$ instead of $\Omega_{1} \mid \Omega_{1}$. Clearly $u_{1}, \cdots, u_{n} \in$ $\mathscr{F}_{\boldsymbol{R}}\left(\Omega_{1} \mid \Omega_{2}\right)$. In this paper we only consider solutions of (generalized) 
word equations in $\mathscr{F}_{\boldsymbol{R}}(\Gamma), \Gamma$ a nonempty set.

DeFINITION 1.2. Let $\nu=\left(a_{1}, \cdots, a_{n}\right), \mu=\left(b_{1}, \cdots, b_{n}\right)$ be solutions of a generalized word equation $\mathscr{W}$ such that $\nu$ has alphabet $\Gamma_{1} \mid \Lambda_{1}$ and $\mu$ has alphabet $\Gamma_{2} \mid \Lambda_{2}$. Then $\nu \leqq \mu$ ( $\mu$ follows from $\left.\nu\right)$ if there exists a natural homomorphism $\psi: \mathscr{F}_{R}\left(\Gamma_{1} \mid \Lambda_{1}\right) \rightarrow \mathscr{F}_{R}\left(\Gamma_{2} \mid \Lambda_{2}\right)$ such that $\psi\left(a_{i}\right)=b_{i}, i=1, \cdots, n$. We write $\psi: \nu \leqq \mu$ if we want to take into account the corresponding homomorphism $\psi$. $u \approx \nu$ if $\mu \leqq \nu \leqq \mu$.

REMARK 1.3. $\leqq$ is transitive in the class of all solutions of a

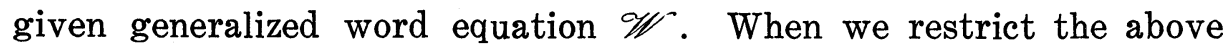
definition to solutions in free semigroups of word equations, we are led to Lentin's concepts in [1] (some of the incorrect concepts in [1] are corrected in [2]).

2. Principal solutions. We now generalize Lentin's concept of a principal solution $[1,2]$.

DeFINITION 2.1. Let $\mu$ be a solution of a generalized word equation $\mathscr{W}^{-}$. Then $\mu$ is a principal solution if for any solution $\nu$ of $\mathscr{W}^{-}, \nu \leqq \mu$ implies $\nu \approx \mu$.

Let $\mu=\left(b_{1}, \cdots, b_{n}\right)$ be a solution of a word equation $\mathscr{W}$. Then $\mu \in \mathscr{S}$ (as a solution of $\mathscr{W}$ ) if there exists a solution $\nu=\left(a_{1}, \cdots, a_{n}\right)$ of $\mathscr{W}$ such that the following properties are true:

(2.2) There exists a unique $f: \nu \leqq \mu$.

(2.3) If $\delta$ is a solution of $\mathscr{W}^{-}$such that $\delta \leqq \mu$, then $\nu \leqq \delta$.

(2.4) Let $\Gamma_{1} \mid \Gamma_{2}$ be the alphabet of $\nu$. Then for each $A \in \Gamma_{1}$, there exists $i \in\{1, \cdots, n\}$ such that $a_{i}$ ends with $A$.

REMARK 2.5. $\nu$ above is necessarily principal. We will show (Theorem 2.19) that if $\mu$ is a solution of any generalized word equation: then $\mu \in \mathscr{S}$. We will also show (Theorem 2.23) that any principal solution $\nu$ satisfies both (2.4) and its right-left dual.

In Lemmas 2.6-2.18 we develop the machinery for replacing the solution of a given word equation with a simpler solution of a related word equation.

LEMMA 2.6. Let $\mathscr{W}^{-}=\left\{w_{1}, w_{2} ; T_{1}, \cdots, T_{s}\right\}$ be a generalized word equation in variables $X_{1}, \cdots, X_{n}, n>1$. Suppose $X_{1}$ is a free variable not appearing in $w_{1} w_{2}$. Let $\mu=\left(u_{1}, \cdots, u_{n}\right)$ be a solution of $\mathscr{W}$. Let $\mathscr{W}^{\prime}=\left\{w_{1}, w_{2} ; T_{1}, \cdots, T_{s}\right\}$ considered as a generalized word equation in variables $X_{2}, \cdots, X_{n}$. Then $\mu^{\prime}=\left(u_{2}, \cdots, u_{n}\right)$ is $a$ solution of $\mathscr{W}^{\prime \prime}$. If $\mu^{\prime} \in \mathscr{S}$, then $\mu \in \mathscr{S}$. 
Proof. Suppose $\mu^{\prime} \in \mathscr{S}$. Correspondingly there exists a solution $\nu^{\prime}$ of $\mathscr{W}$ satisfying (2.2), (2.3), (2.4). Let $\nu^{\prime}=\left(v_{2}, \cdots, v_{n}\right)$ with alphabet $\Gamma^{\prime} \mid \Lambda^{\prime}$. There exists unique $f^{\prime}: \nu^{\prime} \leqq \mu^{\prime}$. Let $A \notin \Gamma^{\prime}$, set $\Gamma=\Gamma^{\prime} \cup\{A\}, \Lambda=\Lambda^{\prime} \cup\{A\}$. Let $v_{1}=A, \nu=\left(v_{1}, v_{2}, \cdots, v_{n}\right)$. Then $\nu$ is a solution of $\mathscr{W}$ and has alphabet $\Gamma \mid \Lambda$. Also $f: \nu \leqq \mu$ where $f$ is an extension of $f^{\prime}$ with $f(A)=u_{1}$. Suppose $g: \nu \leqq \mu$. Let $g^{\prime}$ be the restriction of $g$ to $\mathscr{F}_{R}\left(\Gamma^{\prime} \mid \Lambda^{\prime}\right)$. Then $g^{\prime}: \nu^{\prime} \leqq \mu^{\prime}$. So $f^{\prime}=g^{\prime}$. Since $f(A)=f\left(v_{1}\right)=g\left(v_{1}\right)=g(A), f=g$. Let $B \in \Gamma$. If $B=A$, then $u_{1}$ ends with $A$. Otherwise $B \in \Gamma^{\prime}$ and some $u_{i}, i>1$ ends with $B$. Finally suppose $\delta$ is a solution of $\mathscr{\mathscr { V }}, \delta \leqq \mu$. Let $\delta=\left(a_{1}, \cdots, a_{n}\right)$. Set $\delta^{\prime}=\left(a_{2}, \cdots, a_{n}\right)$. Then $\delta^{\prime}$ is a solution of $\mathscr{W}^{\prime}$. So there exists $g^{\prime}: \nu^{\prime} \leqq \delta^{\prime} . \quad g^{\prime}$ extends to $g: \nu \leqq \delta$ where $g(A)=a_{1}$. This proves that $\mu \in \mathscr{S}$.

Lemma 2.7. Let $\mathscr{Y}=\left\{u w_{1}, u w_{2} ; T_{1}, \cdots, T_{s}\right\}$ be a generalized word equation in variables $X_{1}, \cdots, X_{n}$. Suppose $\mu$ is a solution of $\mathscr{W}$. Then $\mu$ is a solution of $\mathscr{W}^{\prime}=\left\{w_{1}, w_{2} ; T_{1}, \cdots, T_{s}\right\}$ in variables $X_{1}, \cdots, X_{n}$. If $\mu \in \mathscr{S}$ as a solution of $\mathscr{W}^{\prime}$, then $\mu \in \mathscr{S}$ as a solution of $\mathscr{W}$.

Proof. This follows trivially since the solutions of $\mathscr{W}$ are exactly the same as the solutions of $\mathscr{W}^{\prime}$.

Lemma 2.8. Let $\mathscr{W}=\left\{w_{1}, w_{2} ; T_{1}, \cdots, T_{s}\right\}$ be a generalized word equation in variables $X_{1}, \cdots, X_{n}$. Suppose $T_{1}=\left\{X_{1}, \cdots, X_{k}\right\}$, $u, v \in \mathscr{F}\left(T_{1}\right) . \quad$ Let $\mu=\left(b_{1}, \cdots, b_{n}\right), \nu=\left(a_{1}, \cdots, a_{n}\right)$ be solutions of $\mathscr{W}, \nu \leqq \mu . \quad$ Let $c_{1}=u\left(b_{1}, \cdots, b_{k}\right), c_{2}=v\left(b_{1}, \cdots, b_{k}\right), d_{1}=u\left(a_{1}, \cdots, a_{k}\right)$, $d_{2}=v\left(a_{1}, \cdots, a_{k}\right)$. Then $c_{1}=c_{2}$ if and only if $d_{1}=d_{2} ; l\left(c_{1}\right)<l\left(c_{2}\right)$ if and only if $l\left(d_{1}\right)<l\left(d_{2}\right)$.

Proof. Let $f: \nu \leqq \mu$. Let $\Gamma \mid \Lambda$ be the alphabet of $\nu$. There exists $A \in \Gamma$ such that $a_{1}, \cdots, a_{k} \in \bar{A}$. So $c_{1}, c_{2} \in \bar{A}$. Suppose $l\left(c_{1}\right)=l\left(c_{2}\right)$. Then $c_{1}=c_{2}$ and so $d_{1}=f\left(c_{1}\right)=f\left(c_{2}\right)=d_{2}$. Next suppose $l\left(c_{1}\right)<l\left(c_{2}\right)$. Then $c_{2}=c_{1} x$ for some $x \in \mathscr{F}_{R}(\Gamma \mid \Lambda)$. So $d_{2}=f\left(c_{2}\right)=f\left(c_{1}\right) f(x)=d_{1} f(x)$. Thus $l\left(d_{1}\right)<l\left(d_{2}\right)$. Similarly $l\left(c_{2}\right)<l\left(c_{1}\right)$ implies $l\left(d_{2}\right)<l\left(d_{1}\right)$. Since these are mutually exclusive cases, we are done.

LEMMA 2.9. Let $\mathscr{\mathscr { V }}=\left\{u w_{1}, v w_{2} ; T_{1}, \cdots, T_{s}\right\}$ be a generalized word equation in variables $X_{1}, \cdots, X_{n}$ such that $u v \in \mathscr{F}\left(T_{1}\right)$. Suppose $\mu=\left(b_{1}, \cdots, b_{n}\right)$ is a solution of $\mathscr{W}$ such that $u\left(b_{1}, \cdots, b_{n}\right)=$ $v\left(b_{1}, \cdots, b_{n}\right)$. Then $\mu$ is a solution of $\mathscr{W}^{\prime}=\left\{w_{1}, w_{2} ; T_{1}, \cdots, T_{s}\right\}$ in variables $X_{1}, \cdots, X_{n}$. Moreover, if $\mu \in \mathscr{S}$ as a solution of $\mathscr{W}^{\prime}$, then $\mu \in \mathscr{S}$ as a solution of $\mathscr{W}$. 
Proof. Let $\mathscr{A}=\nu \mid \nu$ is a solution of $\mathscr{W}, \nu \leqq \mu\}, \mathscr{B}=\{\nu \mid \nu$ is a solution $\left.\mathscr{W}^{\prime}, \nu \leqq \mu\right\}$. It suffices to show that $\mathscr{A}=\mathscr{B}$. First let $\nu=\left(a_{1}, \cdots, a_{n}\right) \in \mathscr{A}$. Then by Lemma $2.8, u\left(a_{1}, \cdots, a_{n}\right)=v\left(a_{1}, \cdots, a_{n}\right)$ and so $\nu \in \mathscr{B}$. Conversely if $\nu \in \mathscr{B}$, then the same argument shows that $\nu \in \mathscr{A}$.

LEMMA 2.10. Let $\mathscr{W}=\left\{u w_{1}, v X_{j} w_{2} ; T_{1}, \cdots, T_{s}\right\}$ be a generalized word equation in variables $X_{1}, \cdots, X_{n}$ such that $u v \in \mathscr{F}\left(T_{1}\right)$, $X_{j} \in T_{2}$. Let $\mu=\left(b_{1}, \cdots, b_{n}\right)$ be a solution of $\mathscr{W}$ such that $l\left(v\left(b_{1}, \cdots, b_{n}\right)\right)<l\left(u\left(b_{1}, \cdots, b_{n}\right)\right)$. Then $\mu$ is a solution of $\mathscr{W}^{\prime}=\left\{u w_{1}\right.$, $\left.v X_{j} w_{2} ; T_{1} \cup T_{2}, T_{3}, \cdots, T_{s}\right\}$. If $\mu \in \mathscr{S}$ as a solution of $\mathscr{W}^{\prime}$, then $\mu \in \mathscr{S}$ as a solution of $\mathscr{W}$.

Proof. Let $c_{1}=u\left(b_{1}, \cdots, b_{n}\right), c_{2}=v\left(b_{1}, \cdots, b_{n}\right)$. Then $c_{1} \sim c_{2}$, $l\left(c_{2}\right)<l\left(c_{1}\right), c_{1} \cdots=c_{2} b_{j} \cdots$. Since $X_{j}$ is constrained, it follows that $e\left(b_{j}\right)=1$ and $b_{j} \sim c_{1} \sim b_{1}$. So for all $X_{k} \in T_{2}, b_{k} \sim b_{j} \sim b_{1}$. It follows that $\mu$ is a solution of $\mathscr{W}^{\prime}$. It is clear that every solution of $\mathscr{W}^{\prime \prime}$ is a solution of $\mathscr{W}$. So it suffices to show that if $\nu$ is a solution $\mathscr{W}$ and $\nu \leqq \mu$, then $\nu$ is a solution of $\mathscr{W}^{\prime}$. Let $\nu=\left(a_{1}, \cdots, a_{n}\right)$. By Lemma 2.8, $l\left(v\left(a_{1}, \cdots, a_{n}\right)\right)<l\left(u\left(a_{1}, \cdots, a_{n}\right)\right)$ and the above argument shows that $\nu$ is a solution of $\mathscr{W}^{\prime}$.

Lemma 2.11. Let $\mathscr{W}^{\circ}=\left\{w, w ; T_{1}, \cdots, T_{s}\right\}$ be a generalized word equation in variables $X_{1}, \cdots, X_{n}$. Let $\mu=\left(u_{1}, \cdots, u_{n}\right)$ be a solution of $\mathscr{Y}$. Then $\mu \in \mathscr{S}$.

Proof. Since the solutions of $\mathscr{W}$ are the same as those of $\{1$, $\left.1 ; T_{1}, \cdots, T_{s}\right\}$ we can assume without loss of generality that $w=1$. First assume $s=0$. Then by Lemma 2.6, we can assume that $n=1$. Introducing a new symbol $A$ we see that $\nu=(A)$ is a solution of $\mathscr{H}$ and satisfies (2.2), (2.3), and (2.4).

Next assume $s \geqq 1$. Then by Lemma 2.6, we can assume that $\mathscr{W}$ has no free variables. Let $\Omega=\left\{A_{1}, \cdots, A_{n}\right\}$ where $A_{i}=A_{k}$ if and only if $X_{i}, X_{k}$ lie in same $T_{j}$. We define a solution $\nu=\left(v_{1}, \cdots, v_{n}\right)$ of $\mathscr{W}$ as follows: Let $T_{j}=\left\{X_{j(1)}, \cdots, X_{j(t)}\right\}$. Let the alphabet of $\mu=\Gamma_{1} \mid \Gamma_{2}$. There exist $A \in \Gamma_{1}, r_{1}, \cdots, r_{t} \in R^{+}$such that $u_{j(i)}=A^{r_{i}}$. We wish to distinguish two cases. First suppose there exists $d \in \boldsymbol{R}^{+}, k_{1}, \cdots, k_{t} \in Z^{+}$such that $\left(r_{1}, \cdots, r_{t}\right)=d\left(k_{1}, \cdots, k_{t}\right)$. Then we can assume $k_{1}, \cdots, k_{t}$ are relatively prime. Also, if $r_{1}, \cdots, r_{t} \in Z^{+}$, then $d \in Z^{+}$. In such a case set $v_{j(i)}=C^{k_{i}}$ where $C=A_{j(1)}=\cdots=$ $A_{j(t)}, \phi(C)=A^{d}, i=1, \cdots, t$. Next assume there is no such $d$. Then set $v_{j(i)}=A_{j(i)}^{r_{i}}, \phi\left(A_{j(i)}\right)=A, i=1, \cdots, t$. Then $\nu=\left(v_{1}, \cdots, v_{n}\right)$ is a solution of $\mathscr{W}$. Let $\Omega_{1}=\left\{A_{i} \mid A_{i}\right.$ appear integrally in each $v_{j}, j=1$, $\cdots, n\}$. Then $\phi\left(\Omega \backslash \Omega_{1}\right) \subseteq \overline{\Gamma_{1} \backslash \Gamma_{2}}$. Also if $\hat{\phi}$ is the natural extension of $\phi$ 
to $\mathscr{F}_{\mathbf{R}}\left(\Omega \mid \Omega_{1}\right)$, then $\hat{\phi}: \nu \leqq \mu$. Since $e\left(u_{i}\right)=e\left(v_{i}\right)=1$ for all $i$, it is easy to see that $\hat{\phi}$ is unique. Also it is clear that for each $B \in \Omega$ there exists $v_{i}$ such that $v_{i}$ ends with $B$.

Now let $\delta=\left(a_{1}, \cdots, a_{n}\right)$ be a solution of $\mathscr{\mathscr { V }}$, with alphabet $\Lambda_{1} \mid \Lambda_{2}, g: \delta \leqq \mu$. We wish to define $\hat{\psi}: \nu \leqq \delta$. Let $T_{j}=\left\{X_{j(1)}, \cdots, X_{j(t)}\right\}$, $u_{j(i)}=A^{r_{i}}, a_{j(i)}=B^{p_{i}}, i=1, \cdots, t, v_{j(i)}=C^{k_{i}}, C \in \Omega$. Let $\phi(C)=A^{d}$, $g(B)=A^{q}$. Then $d\left(k_{1}, \cdots, k_{t}\right)=\left(r_{1}, \cdots, r_{t}\right)=q\left(p_{1}, \cdots, p_{t}\right)$. Define $\psi(C)=B^{d / q}$. We must show that if $B \in \Lambda_{2}$, then $d / q \in Z^{+}$. So let $B \in \Lambda_{2}$. Then $p_{1}, \cdots, p_{t} \in Z^{+}$. Then by above, $k_{1}, \cdots, k_{t} \in Z^{+}$and are relatively prime. Also $p_{i} / k_{i}=d / q, i=1, \cdots, t$. There exist $\pi_{1}, \cdots, \pi_{t} \in Z$ such that $\pi_{1} k_{1}+\cdots+\pi_{t} k_{t}=1$. So $d / q=\pi_{1} k_{1} d / q+\cdots+$ $\pi_{t} k_{t} d / q=\pi_{1} p_{1}+\cdots+\pi_{t} p_{t} \in Z$. So $d / q \in Z^{+}$. It is clear that if $\hat{\psi}$ is the natural extension of $\psi$ to $\mathscr{F}_{R}\left(\Omega \mid \Omega_{1}\right)$, then $\hat{\psi}: \nu \leqq \delta$. So $\nu$ satisfies (2.2), (2.3) and (2.4); proving the lemma.

Lemma 2.12. Let $\mathscr{W}=\left\{w_{1}, w_{2} ; T_{1}, \cdots, T_{s}\right\}$ be a generalized word equation in variables $X_{1}, \cdots, X_{n}$. Suppose $\mathscr{W}$ has no free variables and $\mu=\left(b_{1}, \cdots, b_{n}\right)$ is a solution of $\mathscr{V}$. Then $\mu \in \mathscr{S}$.

Proof. We prove by induction on $l\left(w_{1} w_{2}\right)+s$. If $w_{1}=w_{2}=1$, then we are done by Lemma 2.11. So assume $w_{1} \neq 1$ (and hence $w_{2} \neq 1$ ). Without loss of generality we can assume that $w_{1}$ starts with a letter in $T_{1}$. We can write $w_{1}=u_{1} u_{2}$ such that $u_{1} \in \mathscr{F}\left(T_{1}\right)$, $l\left(u_{1}\right)$ maximal. Similarly write $w_{2}=v_{1} v_{2}$ such that $v_{1} \in \mathscr{F}\left(T_{1}\right)^{1}, l\left(v_{1}\right)$ maximal. If $u_{1}\left(b_{1}, \cdots, b_{n}\right)=v_{1}\left(b_{1}, \cdots, b_{n}\right)$, then we are done by Lemma 2.9 and the induction hypothesis. So by symmetry assume $l\left(v_{1}\left(b_{1}, \cdots, b_{n}\right)\right)<l\left(u_{1}\left(b_{1}, \cdots, b_{n}\right)\right)$. Let $v_{2}=X_{2} v_{3}$. Without loss of generality we can assume $X_{2} \in T_{2}$. We are again done by the induction hypothesis and Lemma 2.10 .

LemMA 2.13. Let $\mathscr{\mathscr { V }}=\left\{u w_{1}, v w_{2} ; T_{1}, \cdots, T_{s}\right\}$ be a generalized word equation in variables $X_{1}, \cdots, X_{n}$. Let $\mu=\left(b_{1}, \cdots, b_{n}\right)$, $\nu=\left(a_{1}, \cdots, a_{n}\right)$ be solutions of $\mathscr{W}, \nu \leqq \mu$. Set $c_{1}=u\left(a_{1}, \cdots, a_{n}\right), c_{2}=$ $v\left(a_{1}, \cdots, a_{n}\right), d_{1}=u\left(b_{1}, \cdots, b_{n}\right), d_{2}=v\left(b_{1}, \cdots, b_{n}\right)$. Then $c_{1}=c_{2}$ if and only if $d_{1}=d_{2} ; l\left(c_{1}\right)<l\left(c_{2}\right)$ if and only if $l\left(d_{1}\right)<l\left(d_{2}\right)$.

Proof. Let $f: \nu \leqq \mu$. Then $f\left(c_{1}\right)=d_{1}, f\left(c_{2}\right)=d_{2}$. Now $c_{1} \cdots=$ $c_{2} \cdots$. If $l\left(c_{1}\right)=l\left(c_{2}\right)$, then $c_{1}=c_{2}$ and $d_{1}=d_{2}$. If $l\left(c_{1}\right)<l\left(c_{2}\right)$; then $c_{2}=c_{1} c_{2}^{\prime}$ for some $c_{2}^{\prime} \neq 1$ and so $d_{2}=d_{1} d_{2}^{\prime}$ where $d_{2}^{\prime}=f\left(c_{2}^{\prime}\right) \neq 1$. Hence $l\left(d_{1}\right)<l\left(d_{2}\right)$. Similarly $l\left(c_{2}\right)<l\left(c_{1}\right)$ implies $l\left(d_{2}\right)<l\left(d_{1}\right)$. Since these are mutually exclusive cases, we are done.

LeMMA 2.14. Let $\mathscr{Y}=\left\{u w_{1}, v X_{j} w_{2} ; T_{1}, \cdots, T_{s}\right\}$ be a generalized word equation in variables $X_{1}, \cdots, X_{n}$ such that $u v \in \mathscr{F}\left(T_{1}\right)$ 
and $X_{j}$ is a free variable. Let $\mu=\left(b_{1}, \cdots, b_{n}\right)$ be a solution of $\mathscr{W}$. Set $c_{1}=u\left(b_{1}, \cdots, b_{n}\right), c_{2}=v\left(b_{1}, \cdots, b_{n}\right)$. Suppose $l\left(c_{2} b_{j}\right) \leqq l\left(c_{1}\right) . \quad$ Let $\mathscr{W}^{\prime}=\left\{u w_{1}, v X_{j} w_{2} ; T_{1} \cup\left\{X_{j}\right\}, T_{2}, \cdots, T_{s}\right\}$ in variables $X_{1}, \cdots, X_{n}$. Then $\mu$ is a solution of $\mathscr{W}^{\prime}$. If $\mu \in \mathscr{S}$ as a solution of $\mathscr{W}^{\prime}$, then $\mu \in \mathscr{S}$ as a solution of $\mathscr{Y}$.

Proof. $l\left(c_{2} b_{j}\right) \leqq l\left(c_{1}\right)$ and $c_{1} \cdots=c_{2} b_{j} \cdots$, and so $e\left(b_{j}\right)=1, b_{j} \sim c_{1}$. It follows that $\mu$ is a solution of $\mathscr{W}^{\prime}$. It is also clear that every solution of $\mathscr{W}^{\prime}$ is a solution of $\mathscr{W}$. We need only show that if $\nu$ is a solution of $\mathscr{W}$ and $\nu \leqq \mu$, then $\nu$ is a solution of $\mathscr{W}^{\prime}$. So let $\nu=\left(a_{1}, \cdots, a_{n}\right) \leqq \mu$. Let $d_{1}=u\left(a_{1}, \cdots, a_{n}\right), d_{2}=v\left(a_{1}, \cdots, a_{n}\right)$. Then by Lemma $2.13, l\left(d_{2} a_{j}\right) \leqq l\left(a_{1}\right)$. So by the above argument $\nu$ is a solution of $\mathscr{W}^{\prime}$.

Lemma 2.15. Let $\mathscr{\mathscr { V }}=\left\{u w_{1}, v X_{j} w_{2} ; T_{1}, \cdots, T_{s}\right\}$ be a generalized word equation in variables $X_{1}, \cdots, X_{n}$ such that $u v \in \mathscr{F}\left(T_{1}\right)$ and $X_{j}$ is a free variable. Let $\mu=\left(b_{1}, \cdots, b_{n}\right)$ be a solution of $\mathscr{W}$. Set $c_{1}=u\left(b_{1}, \cdots, b_{n}\right), c_{2}=v\left(b_{1}, \cdots, b_{n}\right)$. Suppose $l\left(c_{2} b_{j}\right)>l\left(c_{1}\right)>l\left(c_{2}\right)$. Then there exist $b_{n+1}, b_{j}^{\prime} \neq 1$ such that $b_{n+1} \sim c_{1} \sim c_{2}, b_{j}=b_{n+1} b_{j}^{\prime}$ and $c_{1}=c_{2} b_{n+1}$. Introduce new variables $X_{j}^{\prime}, X_{n+1}$. Set $T_{1}^{\prime}=T_{1} \cup\left\{X_{n+1}\right\}$. Let $w_{1}^{\prime}, w_{2}^{\prime}$ be the words obtained by replacing $X_{j}$ by $X_{n+1} X_{j}^{\prime}$ in $w_{1}, w_{2}$ respectively. Let $\mathscr{W}^{\prime}$ be the generalized word equation $\left\{w_{1}^{\prime}, X_{j}^{\prime} w_{2}^{\prime}\right.$; $\left.T_{1}^{\prime}, T_{2}, \cdots, T_{s}\right\}$ in variables $X_{1}, \cdots X_{j-1}, X_{j}^{\prime}, X_{j+1}, \cdots, X_{n}, X_{n+1}$. Then $\mu^{\prime}=\left(b_{1}, \cdots, b_{j-1}, b_{j}^{\prime}, b_{j+1}, \cdots, b_{n}, b_{n+1}\right)$ is a solution $\mathscr{W}^{\prime}$. Moreover, if $\mu^{\prime} \in \mathscr{S}$, then $\mu \in \mathscr{S}$.

Proof. Now $c_{1} \cdots=c_{2} b_{j} \cdots$ and so the existence of $b_{n+1}, b_{j}^{\prime}$ follows. It is clear that $\mu^{\prime}$ is a solution of $\mathscr{Y}^{\prime}$. Suppose $\mu^{\prime} \in \mathscr{S}$. Then there exists a solution $\nu^{\prime}=\left(a_{1}, \cdots, a_{j-1}, a_{j}^{\prime}, a_{j+1}, \cdots, a_{n}, a_{n+1}\right)$ of $\mathscr{W}^{\prime}$, $f: \nu^{\prime} \leqq \mu^{\prime}$ such that (2.2), (2.3), (2.4) are true. Let $a_{j}=a_{n+1} a_{j}^{\prime}, \nu=$ $\left(a_{1}, \cdots, a_{j-1}, a_{j}, a_{j+1}, \cdots, a_{n}\right)$. By Lemma $2.8, \nu^{\prime}$ is a solution of the word equation $\left\{u, v X_{n+1}\right\}$. It now follows easily that $\nu$ is a solution of $\mathscr{W}$. Let $\Gamma \mid \Lambda=$ alphabet of $\nu^{\prime}$. Then alphabet of $\nu=\Gamma \mid \Lambda$ and $f: \nu \leqq \mu$. If $g: \nu \leqq \mu$, then by Lemma $2.13 g: \nu^{\prime} \leqq \mu^{\prime}$ and so $g=f$. Let $A \in \Gamma$. If $a_{n+1}$ ends with $A$, then so does $a_{k}$ where $X_{k} \in T_{1}$. If $a_{j}^{\prime}$ ends with $A$, then so does $a_{j}$. It follows that $\nu$ satisfies (2.4). Finally let $\delta=\left(d_{1}, \cdots, d_{n}\right)$ be a solution of $\mathscr{W}^{-}, h: \delta \leqq \mu$. Let $c_{3}=u\left(d_{1}, \cdots, d_{n}\right)$, $c_{4}=v\left(d_{1}, \cdots, d_{n}\right)$. By Lemma 2.13, $l\left(c_{4} d_{j}\right)>l\left(c_{3}\right)>l\left(c_{4}\right)$. As above there exist $d_{n+1}, d_{j}^{\prime} \neq 1$, such that $d_{n+1} \sim c_{3} \sim c_{4}, d_{j}=d_{n+1} d_{j}^{\prime}, c_{3}=c_{4} d_{n+1}$. It follows that $\delta^{\prime}=\left(d_{1}, \cdots, d_{j-1}, d_{j}^{\prime}, d_{j+1}, \cdots, d_{n}, d_{n+1}\right)$ is a solution $\mathscr{W}^{\prime}$. It is also easily seen that $h: \delta^{\prime} \leqq \mu^{\prime}$. So there exist $h_{1}: \nu^{\prime} \leqq \delta^{\prime}$. It follows as above that $h_{1}: \nu \leqq \delta$. Thus $\mu \in \mathscr{S}$, proving the lemma.

Lemma 2.16. Let $\mathscr{\mathscr { V }}=\left\{w_{1}, w_{2} ; T_{1}, \cdots, T_{s}\right\}$ be a generalized 
word equation in variables $X_{1}, \cdots, X_{n}$. Suppose $X_{j}$ is a free variable of $\mathscr{W}$. Let $\mu=\left(b_{1}, \cdots, b_{n}\right)$ be a solution $\mathscr{W}$ such that for any solution $\nu=\left(a_{1}, \cdots, a_{n}\right)$ of $\mathscr{W}, \nu \leqq \mu$ implies $e\left(a_{j}\right)=1$. Let $\mathscr{W}^{\prime}$ be the generalized word equation $\left\{w_{1}, w_{2} ; T_{1}, \cdots T_{s},\left\{X_{j}\right\}\right\}$ in variables $X_{1}, \cdots, X_{n}$. Then $\mu$ is a solution of $\mathscr{W}^{\prime}$. If $\mu \in \mathscr{S}$ as a solution of $\mathscr{\ell}^{\prime}$, then $\mu \in \mathscr{S}$ as a solution of $\mathscr{W}$.

Proof. Clearly, every solution of $\mathscr{W}^{\prime}$ is a solution of $\mathscr{W}$. Let $\nu$ be a solution of $\mathscr{W}$ such that $\nu \leqq \mu$. Then by hypothesis $\nu$ is a solution $\mathscr{W}^{\prime}$. Then lemma now follows trivially.

Lemma 2.17. Let $\mathscr{W}=\left\{X_{i} w_{1}, X_{j} w_{2} ; T_{1}, \cdots, T_{s}\right\}$ be a generalized word equation in variable $X_{1}, \cdots, X_{n}$ such that $X_{j}$ is a free variable. Let $\mu=\left(b_{1}, \cdots, b_{n}\right)$ be a solution of $\mathscr{W}^{-}$such that $l\left(b_{i}\right)<l\left(b_{j}\right)$. Then there exist $b_{j}^{\prime} \neq 1$ such that $b_{j}=b_{i} b_{j}^{\prime}$. Introduce a new variable $X_{j}^{\prime}$ and let $w_{1}^{\prime}, w_{2}^{\prime}$ be the words obtained by replacing $X_{j}$ by $X_{i} X_{j}^{\prime}$. Let $\mathscr{W}^{\prime}=\left\{w_{1}^{\prime}, X_{j}^{\prime} w_{2}^{\prime} ; T_{1}, \cdots, T_{s}\right\}$ in variables $X_{1}, \cdots, X_{n}$. Then $\mu^{\prime}=\left(b_{1}, \cdots, b_{j-1}, b_{j}^{\prime}, b_{j+1}, \cdots, b_{n}\right)$ is a solution of $\mathscr{W}^{\prime}$. Let $\mathscr{A}=\{\nu \mid \nu$ is a solution of $\mathscr{W}, \nu \leqq \mu\}, \mathscr{B}=\left\{\nu^{\prime} \mid \nu^{\prime}\right.$ is a solution of $\left.\mathscr{W}^{\prime}, \nu^{\prime} \leqq \mu^{\prime}\right\}$. Let $\nu=\left(a_{1}, \cdots, a_{n}\right) \in \mathscr{A}$. Then there exists $a_{j}^{\prime} \neq 1$ such that $a_{j}=a_{i} a_{j}^{\prime}$. If $\Phi(\nu)$ is obtained by replacing $a_{j}$ by $a_{j}^{\prime}$ in $\nu$, then $\Phi(\nu) \in \mathscr{B}$. If $f: \nu \leqq \mu$, then $f: \Phi(\nu) \leqq \mu^{\prime}$. Let $\nu^{\prime}=\left(a_{1}, \cdots, a_{j}^{\prime}\right.$, $\left.\cdots, a_{n}\right) \in \mathscr{B}$. If $\Psi(\nu)$ is obtained by replacing $a_{j}^{\prime}$ by $a_{j}=a_{i} a_{j}^{\prime}$, then $\Psi\left(\nu^{\prime}\right) \in \mathscr{A}$. If $f: \nu^{\prime} \leqq \mu^{\prime}$, then $f: \Psi\left(\nu^{\prime}\right) \leqq \mu$. Moreover $\Psi=\Phi^{-1}$. Finally $\mu \in \mathscr{S}$ if and only if $\mu^{\prime} \in \mathscr{S}$.

Proof. Suppose $\nu=\left(a_{1}, \cdots, a_{n}\right) \in \mathscr{A}$. By Lemma 2.13, $l\left(a_{i}\right)<l\left(a_{j}\right)$ and so there exists $a_{j}^{\prime} \neq 1$ such that $a_{j}=a_{i} a_{j}^{\prime}$. So $\Phi$ makes sense. Let $\nu^{\prime}=\Phi(\nu)$. It is clear that $\nu$ satisfies (2.4) if and only if $\nu^{\prime}$ does. Rest of the assertions are now fairly obvious.

Lemma 2.18. Let $\mathscr{W}=\left\{X_{1} w_{1}, X_{2} w_{2} ; T_{1}, \cdots, T_{s}\right\}$ be a generalized word equation in variables $X_{1}, \cdots, X_{n}$ such that $X_{1}, X_{2}$ are free variables. Suppose $\mu=\left(b_{1}, \cdots, b_{n}\right)$ is a solution of $\mathscr{W}$. Suppose $b_{1}=b_{2}$. Let $w_{1}^{\prime}, w_{2}^{\prime}$ be obtained by replacing $X_{1}$ by $X_{2}$ in $w_{1}, w_{2}$, respectively. Let $\mathscr{W}^{\prime}$ be the generalized word equation $\left\{w_{1}^{\prime}, w_{2}^{\prime}\right.$; $\left.T_{1}, \cdots, T_{s}\right\}$ in variables $X_{2}, \cdots, X_{n}$. Then $\mu^{\prime}=\left(b_{2}, \cdots, b_{n}\right)$ is a solution of $\mathscr{W}^{\prime}$. Moreover, if $\mu^{\prime} \in \mathscr{S}$, then $\mu \in \mathscr{S}$.

Proof. Let $\mathscr{A}=\{\nu \mid \nu$ is a solution of $\mathscr{W}, \nu \leqq \mu\}, \mathscr{B}=\{\nu \mid \nu$ is a solution of $\left.\mathscr{W}^{\prime}, \nu \leqq \mu^{\prime}\right\}$. If $\nu=\left(a_{1}, \cdots, a_{n}\right) \in \mathscr{A}$, then by Lemma $2.13, a_{1}=a_{2}$. So $\Phi(\nu)=\left(a_{2}, \cdots, a_{n}\right) \in \mathscr{B}$. Also, $f: \nu \leqq \mu$ implies $f: \Phi(\nu) \leqq \Phi(\mu)=\mu^{\prime}$. It is also clear that $\nu$ satisfies (2.4) if and only if $\Phi(\nu)$ does. If $\nu^{\prime}=\left(a_{2}, \cdots, a_{n}\right) \in \mathscr{B}$, then let $\Phi\left(\nu^{\prime}\right)=\nu=\left(a_{2}, a_{2}, \cdots, a_{n}\right)$. 
It is clear that $\nu \in \mathscr{A}$. Also $g: \nu^{\prime} \leqq \mu^{\prime}$ implies $g: \nu \leqq \mu$. Finally, it is obvious that $\Phi \Psi, \Psi \Phi$ are identity maps of $\mathscr{B}, \mathscr{A}$ respectively. So $\Psi=\Phi^{-1}$. This correspondence yields the result.

THeOREM 2.19. Let $\mathscr{W}=\left\{w_{1}, w_{2} ; T_{1}, \cdots, T_{s}\right\}$ be a generalized word equation in variables $X_{1}, \cdots, X_{n}$ and let $\mu=\left(b_{1}, \cdots, b_{n}\right)$ be a solution of $\mathscr{W}^{-}$. Then $\mu \in \mathscr{S}$.

Proof. Let $d$ denote the number of free variables of $\mathscr{Y}$. We prove by induction on $d$. If $d=0$, we are done by Lemma 2.12. So assume $d>0$. We now (for a given $d$ ) proceed by induction on $s$. If $w_{1}=w_{2}$, we are done by Lemma 2.11. So assume $w_{1} \neq w_{2}$. Let $w_{1}$ start with $X_{i}, w_{2}$ start with $X_{j}$. By Lemma 2.7, we can assume that $X_{i} \neq X_{j}$. Next suppose $X_{i}, X_{j}$ are both constrained, $X_{i} \in T_{1}$. If $X_{j} \notin T_{1}$, we are done by Lemma 2.10 and our induction hypothesis on $s$. So let $X_{j} \in T_{1}$. Now $w_{1}=u w_{3}, w_{2}=v w_{4}$ such that $u, v \in \mathscr{F}\left(T_{1}\right), l(u v)$ maximal. Let $c_{1}=u\left(b_{1}, \cdots, b_{n}\right), c_{2}=v\left(b_{1}, \cdots, b_{n}\right)$. By Lemma 2.9 and an easy induction on $l\left(w_{1} w_{2}\right)$, we can assume that $l\left(c_{1}\right) \neq l\left(c_{2}\right)$. By symmetry assume $l\left(c_{2}\right)<l\left(c_{1}\right)$. If $w_{4}$ starts with a constrained variables we are done by Lemma 2.10 and our induction hypothesis on $s$. So assume $w_{4}$ starts with a free variable. Thus by Lemmas 2.14 and 2.15, we can assume, without loss of generality, that either $X_{i}$ or $X_{j}$ is free. By Lemma 2.6 and our induction hypothesis on $d$, we can assume that each free variable occurs in $w_{1} w_{2}$.

We now assume $u \in \mathscr{S}$ and obtain a contradiction. Let $\mathscr{C}=$ $\left\{r \mid X_{r} \in T_{q}\right.$ for some $\left.q\right\}$. Let $\mu^{(0)}=\mu, b_{r}^{(0)}=b_{r}, r=1, \cdots, n$, $\mathscr{W}^{(0)}=\mathscr{W}$, $w_{1}^{(0)}=w_{1}, w_{2}^{(0)}=w_{2}, X_{r}^{(0)}=X_{r}, r=1, \cdots, n$. Also let $\mathscr{Q}^{(0)}=\left\{\boldsymbol{\nu}_{\alpha}^{(0)}=\right.$ $\left.\left(a_{1}^{\alpha, 0}, \cdots, a_{n}^{\alpha, 0}\right) \mid \alpha \in \Omega\right\}$ denote the class of all solutions of $\mathscr{W}$ such that $\nu \leqq \mu$. Let $u^{(0)}=w_{1} w_{2}\left(X_{1}, \cdots, X_{n}\right), m_{r}^{(0)}$ the number of times $X_{r}$ appears in $u^{(0)}$. Then $m_{r}^{(0)}>0$ for $r \notin \mathscr{C}$. Let $b=u^{(0)}\left(b_{1}, \cdots, b_{n}\right)$ and for $\alpha \in \Omega$, let $a_{\alpha}=u^{(0)}\left(a_{1}^{\alpha, 0}, \cdots, a_{n}^{\alpha, 0}\right)$. We will construct a sequence of generalized word equations $\mathscr{W}^{(k)}$ with particular solutions $\mu^{(k)}=$ $\left(b_{1}^{(k)}, \cdots, b_{n}^{j k)}\right)$ such that the following conditions are true for all $k \in Z$. Intuitively we are looking at the sequence of word equations obtained by always truncating on the left.

(I) $\mathscr{\mathscr { W }}^{(k)}=\left\{w_{1}^{(k)}, w_{2}^{(k)} ; T_{1}, \cdots, T_{s}\right\}$ in variables $X_{1}^{(k)}, \cdots, X_{n}^{(k)}$ such that $X_{r}^{(k)}=X_{r}$ for $r \in \mathscr{C}$.

(II) $w_{1}^{(k)}, w_{2}^{(k)}$ start with different variables; at least one of which is free.

(III) The class of solutions $\nu$ of $\mathscr{W}^{(k)}$ such that $\nu \leqq \mu^{(k)}$ can be written as $\mathscr{Z}^{(k)}=\left\{\nu_{\alpha}^{(k)}=\left(a_{1}^{\alpha, k}, \cdots, a_{n}^{\alpha, k}\right) \mid \alpha \in \Omega\right\}$.

(IV) There exists $u^{(k)} \in \mathscr{F}\left(X_{1}, \cdots, X_{n}\right)$ such that $b=$ $u^{(k)}\left(b_{1}^{(k)}, \cdots, b_{n}^{(k)}\right), a_{\alpha}=u^{(k)}\left(a_{1}^{\alpha, k}, \cdots, a_{n}^{\alpha, k}\right)$ for all $\alpha \in \Omega$. Let $m_{r}^{(k)}$ denote the number of times $X_{r}$ appears in $u^{(k)}$. If $k>0$, then $m_{r}^{(k)} \geqq m_{r}^{(k-1)}$, 
$r=1, \cdots, n$ and $\sum_{r=1}^{n} m_{r}^{(k)}>\sum_{r=1}^{n} m_{r}^{(k-1)}$.

(V) If $k>0$, then there exist $u_{1}^{(k)}, \cdots, u_{n}^{(k)} \in \mathscr{F}\left(X_{1}, \cdots, X_{n}\right)$ such that $b_{r}^{(k-1)}=u_{r}^{(k)}\left(b_{1}^{(k)}, \cdots, b_{n}^{(k)}\right), a_{r}^{\alpha, k-1}=u_{r}^{(k)}\left(a_{1}^{\alpha, k}, \cdots, a_{n}^{\alpha, k}\right), r=1, \cdots, n$, $\alpha \in \Omega$.

(VI) Suppose $k>0$. Then there exists $q \in\{1, \cdots, n\} q \notin \mathscr{C}$, such that $X_{q}^{(k)} \neq X_{q}^{(k-1)}$ and $X_{r}^{(k)}=X_{r}^{(k-1)}$ for all $r \in\{1, \cdots, n\}, r \neq q$. Also there exists $t \in\{1,2\}$ such that $w_{t}^{(k)}$ starts with $X_{q}^{(k)}$ and $w_{t}^{(k-1)}$ starts with $X_{q}^{(k-1)}$. If $w_{3-t}^{(k-1)}$ starts with $X_{p}^{(k-1)}$, then $m_{p}^{(k)}>m_{p}^{(k-1)}$.

(VII) Suppose $k>0, r \in\{1, \cdots, n\}$. If $X_{r}^{(k)}=X_{r}^{(k-1)}$, then $b_{r}^{(k)}=b_{r}^{(k-1)}, \alpha_{r}^{\alpha, k}=a_{r}^{\alpha, k-1}$ for all $\alpha \in \Omega$. If $X_{r}^{(k)} \neq X_{r}^{(k-1)}$, then $\left.b_{r}^{(k)}\right|_{f} b_{r}^{(k-1)}$, $a_{r}^{\alpha, k}{ }_{f} a_{r}^{\alpha, k-1}$ for all $\alpha \in \Omega$.

(VIII) $\mu^{(k)} \notin \mathscr{S}$.

Clearly $\mathscr{W}^{(0)}$ satisfies (I) to (VIII). We proceed by induction. So, having constructed $\mathscr{W}^{(0)}, \cdots, \mathscr{W}^{(k)}$ satisfying (I) to (VIII), we proceed to construct $\mathscr{W}^{(k+1)}$. Let $w_{1}^{(k)}=X_{p}^{(k)} w_{1}^{\prime}, w_{2}^{(k)}=X_{q}^{(k)} w_{2}^{\prime}$. Then $p \neq q$ and either $X_{p}^{(k)}$ or $X_{q}^{(k)}$ is free. $\mu^{(k)}=\left(b_{1}^{(k)}, \cdots, b_{n}^{(k)}\right)$ is a solution of $\mathscr{W}^{(k)}$. If $l\left(b_{p}^{(k)}\right)=l\left(b_{q}^{(k)}\right)$ then $b_{p}^{(k)}=b_{q}^{(k)}$ and by Lemmas 2.14 and 2.18, $\mu^{(k)} \in \mathscr{S}$, contradicting (VIII). By symmetry assume $l\left(b_{p}^{(k)}\right)<l\left(b_{q}^{(k)}\right)$. By Lemma 2.14, $X_{q}^{(k)}$ is free. Also $b_{q}^{(k)}=b_{p}^{(k)} b_{q}^{(k+1)}$ for some $b_{q}^{(k+1)} \neq 1$. Introduce a new variable $X_{q}^{(l k+)}$ and set $X_{r}^{(k+1)}=$ $X_{r}^{(k)}$ for $r \neq q$. Also let $b_{r}^{(k+1)}=b_{r}^{(k)}$ for $r \neq q, \mu^{(k+1)}=\left(b_{1}^{(k+1}, \cdots, b_{n}^{(k+1)}\right)$. Let $w_{1}^{(k+1)}$ be the word obtained by replacing $X_{q}^{(k)}$ by $X_{p}^{(k)} X_{q}^{(k+1)}$ in $w_{1}^{\prime}$ and $w_{2}^{(k+1)}=X_{q}^{(k+1)} w_{2}^{\prime \prime}$ where $w_{2}^{\prime \prime}$ is the word obtained by replacing $X_{q}^{(k)}$ by $X_{p}^{(k)} X_{q}^{(k+1)}$ in $w_{2}^{\prime}$. Then $w_{1}^{(k+1)}$ does not start with $X_{q}^{(k+1)}$. Let $\mathscr{\mathscr { V }}^{(k+1)}=\left\{w_{1}^{(k+1)}, w_{2}^{(k+1)} ; T_{1}, \cdots, T_{s}\right\}$ be the generalized word equation in variables $X_{1}^{(k+1)}, \cdots, X_{n}^{(k+1)}$. So (I), (II) are satisfied. By Lemma 2.17, $\mu^{(k+1)}$ is a solution $\mathscr{W}^{(k+1)}$ and $\mu^{(k+1)} \notin \mathscr{S}$. So (VIII) is satisfied. If $\alpha \in \Omega$, then by Lemma 2.17, $a_{q}^{\alpha, k}=a_{p}^{\alpha, k}=a_{p}^{\alpha, k} a_{q}^{\alpha, k+1}$ for some $a_{q}^{\alpha, k+1} \neq 1$. Set $a_{r}^{\alpha, k}=a_{r}^{\alpha, k+1}$ for $r \neq q, \nu_{\alpha}^{(k+1)}=\left(a_{1}^{\alpha, k+1}, \cdots, a_{n}^{\alpha, k+1}\right)$. Let $\mathscr{Q}^{(k+1)}=\left\{\nu_{\alpha}^{(k+1)} \mid \alpha \in \Omega\right\}$. That $\mathscr{X}^{(k+1)}$ satisfies (III) follows from Lemma 2.17. Let $u^{(k+1)}$ be the word obtained by replacing $X_{q}$ by $X_{p} X_{q}$ in $u^{(k)}$. Let $m_{r}^{(k+1)}$ denote the number of times $X_{r}^{(k)}$ appears in $u^{(k+1)}, r=1, \cdots, n$. Then $m_{r}^{(k)}=m_{r}^{(k+1)}$ for $r \neq p$ and $m_{p}^{(k+1)}=m_{p}^{(k)}+m_{q}^{(k)} \geqq m_{p}^{(k)}+m_{q}^{(0)}>m_{p}^{(k)}$. It is now easy to see that (IV), (VI), (VII) are satisfied. Let $u_{r}^{(k+1)}=$ $X_{r}$ for $r \neq q, u_{q}^{(k+1)}=X_{p} X_{q}$. It is then clear that (V) is satisfied. This completes the construction.

Let $\mathscr{B}=\left\{r \mid m_{r}^{(k)} \rightarrow \infty\right.$ as $\left.k \rightarrow \infty\right\} . \quad$ By (IV), $\sum_{\mid r=1}^{n} m_{r}^{(k)} \rightarrow \infty$ and so $\mathscr{B} \neq \varnothing$. Let $\mathscr{A}=\left\{r \mid\right.$ for any $k \in Z^{+}$, there exists $k^{\prime}>k$ such that $\left.X_{r}^{(k \prime)} \neq X_{r}^{(k r+1)}\right\}$. We claim that $\mathscr{B}=\mathscr{A}$. First assume $r \in \mathscr{B}$. It follows from (IV) that $l\left(b_{r}^{(k)}\right) \rightarrow 0$ as $k \rightarrow \infty$. It then follows from (VII) that $r \in \mathscr{A}$. Hence $\mathscr{B} \cong \mathscr{A}$. Conversely let $r \in \mathscr{A}$. Suppose $r \notin \mathscr{B}$. Then there exists $k \in Z^{+}$such that $m_{r}^{(k)}=m_{r}^{\left(k^{\prime}\right)}$ for all $k^{\prime}>k$. There exists $m>k$ such that $X_{r}^{(m)} \neq X_{r}^{(m+1)}$. There exists $\pi \in \mathscr{B}$. So $\pi \neq r$ and by above, $\pi \in \mathscr{A}$. So there exists $\theta \in Z^{+}, \theta>m$ such 
that $X_{r}^{(\theta)} \neq X^{(\theta+1)}$. By (VI) $X_{r}^{(\theta)}=X_{r}^{(\theta+1)}$. Choose $j>m$, minimal so that $X_{r}^{(j)}=X_{r}^{(j+1)}$. Then $X_{r}^{(j-1)} \neq X_{r}^{(j)}$. By (VI), there exists $t \in\{1,2\}$ such that $w_{t}^{(j)}$ starts with $X_{r}^{(j)}$. It then follows again by (VI) that $m_{r}^{(j+1)}>m_{r}^{(j)}$, a contradiction. So $r \in \mathscr{B}$ and $\mathscr{A}=\mathscr{B}$. It follows that $\mathscr{B} \cap \mathscr{C}=\varnothing$.

Since $\mathscr{A}=\mathscr{B}$, it follows from (VII) that there exists $\varepsilon \in \boldsymbol{R}^{+}$ such that $l\left(b_{r}^{(k)}\right) \geqq \varepsilon$ for all $k \in Z^{+}, r \in\{1, \cdots, n\} \backslash \mathscr{B}$. By (IV), it follows that $l\left(b_{r}^{(k)}\right) \rightarrow 0$ for all $r \in \mathscr{B}$. Since $\mathscr{B}$ is finite, there exists $M \in Z^{+}$such that $l\left(b_{r}^{(M)}\right)<\varepsilon$ for all $r \in \mathscr{B}$. Let $m>M$. Repeated application $(\mathrm{V})$ shows that there exist $v_{1}^{(m)}, \cdots, v_{n}^{(m)} \in \mathscr{F}\left(X_{1}, \cdots, X_{n}\right)$ such that $a_{r}^{\alpha, M}=v_{r}^{(m)}\left(a_{1}^{\alpha, m}, \cdots, a_{n}^{\alpha, m}\right), r=1, \cdots, n, \alpha \in \Omega$ and $b_{r}^{(M)}=$ $v_{r}^{(m)}\left(b_{1}^{(m)}, \cdots, b_{n}^{(m)}\right), r=1, \cdots, n$. The choice of $M$ implies that for $r \in \mathscr{B}, v_{r}^{(m)}$ does not involve $X_{t}$ for any $t \notin \mathscr{B}$. Thus for $r \in \mathscr{B}$, $v_{r}^{(m)}$ is a word in $X_{t}(t \in \mathscr{B})$. Let $\alpha \in \Omega, r \in \mathscr{B}$. We will show that $e\left(a_{r}^{\alpha, M}\right)=1$. Since $\mathscr{A}=\mathscr{B}$, we see by (VI) that there exists $N>M$ such that for any $\theta>N, w_{1}^{(\theta)}, w_{2}^{(\theta)}$ do not start with $X_{j}^{(\theta)}$ for any $j \notin \mathscr{B}$. It follows from (IV) that there exists $L>N$ such that for any $\theta \geqq L, t \in \mathscr{B}, e\left(a_{t}^{\alpha, \theta}\right)=1$. Since $r \in \mathscr{A}$, there exists $P>N$ such that $X_{r}^{(P)} \neq X_{r}^{(P+1)}$. So by (VI), there exists $\pi \in\{1,2\}$, such that $w_{\pi}^{(P)}$ starts with $X_{r}^{(P)}$. Suppose $w_{3-\pi}^{(P)}$ starts with $X_{j}^{(P)}$. Then by above $j \in \mathscr{B}$. So $e\left(a_{r}^{\alpha, P}\right)=e\left(a_{j}^{\alpha, P}\right)=1$ and so $a_{j}^{\alpha, P} \sim a_{r}^{\alpha, P}$. We claim that for any $p \geqq P$, if either $w_{1}^{(p)}$ or $w_{2}^{(p)}$ starts with $X_{t}^{(p)}$, then $a_{t}^{\alpha, p} \sim a_{r}^{\alpha, P}$. We prove the above property for $p+1$, assuming it to be true for $p$. By symmetry, let $w_{1}^{(p)}$ start with $X_{t}^{(p)}, X_{t}^{(p)} \neq X_{t}^{(p+1)}$. Then by (VI) $w_{1}^{(p+1)}$ starts with $X_{t}^{(p+1)}$ and by (VII), $a_{t}^{\alpha, p+1} \sim a_{t}^{\alpha, p} \sim a_{r}^{\alpha, p}$. If $w_{2}^{(p+1)}$ starts with $X_{q}^{(p+1)}$, then clearly $a_{q}^{\alpha, p+1} \sim a_{t}^{\alpha, p+1} \sim a_{r}^{\alpha, P}$. Thus the asserted claim is true. Now let $t \in \mathscr{B}$. Then since $t \in \mathscr{A}$, there exists $p>P$ such that $X_{t}^{(p)} \neq X_{t}^{(p)}$. So by (VI), either $w_{1}^{(p)}$ or $w_{2}^{(p)}$ starts with $X_{t}^{(p)}$. By above, $a_{t}^{\alpha, p} \sim a_{r}^{\alpha, p}$. Since $p>L$ and $e\left(a_{t}^{\alpha, L}\right)=1$, we see by (VII) that $a_{t}^{\alpha, L} \sim a_{r}^{\alpha, P}$. Now $a_{r}^{\alpha, M}=v_{r}^{(L)}\left(a_{1}^{\alpha, L}, \cdots, a_{n}^{\alpha, L}\right)$. Since $X_{t}$ does not appear in $v_{r}^{(L)}$ for $t \notin \mathscr{B}$, we see by the above that $e\left(a_{r}^{\alpha, M}\right)=1$. Since $M$ is independent of $\alpha$, we see by Lemma 2.16 (and our induction hypothesis on $d$ ) that $\mu^{(\alpha)} \in \mathscr{S}$, contradicting (VIII). This contradiction proves the theorem.

CoROLLARY 2.20. Let $\mathscr{W}$ be a generalized word equation and $\mu, \nu_{1}, \nu_{2}$ solutions of $\mathscr{W}$ such that $\nu_{1} \leqq \mu, \nu_{2} \leqq \mu$. Then there exists a solution $\nu$ of $\mathscr{W}$ such that $\nu \leqq \nu_{1}$ and $\nu \leqq \nu_{2}$. In particular, if $\nu_{1}, \nu_{2}$ are principal, then $\nu_{1} \approx \nu_{2}$.

LEMMA 2.21. Let $\mathscr{W}$ be a generalized word equation, $\mu a$ principal solution of $\mathscr{W}$ such that $\mu$ satisfies (2.4). Then $\mu$ satisfies the right-left dual of (2.4).

Proof. By the dual of Theorem 2.19, there exists a principal 
solution $\nu$ of $\mathscr{W}$ satisfying the dual of (2.4) such that $\nu \leqq \mu$. Since $\mu$ is principal, $\nu \approx \mu$. There exist $f: \nu \leqq \mu, g: \mu \leqq \nu$. Let the alphabet of $\mu=\Gamma_{1} \mid \Lambda_{1}$, alphabet of $\nu=\Gamma_{2} \mid \Lambda_{2}$. Then $f\left(\Gamma_{2} \mid \Lambda_{2}\right) \cong \overline{\Gamma_{1} \backslash \Lambda_{1}}$, $g\left(\Gamma_{1} \backslash \Lambda_{1}\right) \cong \widetilde{\Gamma_{2} \backslash \Lambda_{2}}$. Let $\nu=\left(a_{1}, \cdots, a_{n}\right), \mu=\left(b_{1}, \cdots, b_{n}\right)$. Let $A \in \Lambda_{1}$. Then there exists $i \in\{1, \cdots, n\}, b \in \mathscr{F}_{\mathbf{R}}\left(\Gamma_{1} \mid \Lambda_{1}\right)^{1}$ such that $b_{i}=b A$. So $a_{i}=g\left(b_{i}\right)=a g(A)$ where $a=g(b)$. Let $g\left(b_{i}\right)$ end with $B \in \Gamma_{2}$. We claim that $B \in \Lambda_{2}$. Suppose not. Now $g(A)=c B^{r}$ for some $r \in \boldsymbol{R}^{+}$, $c \in \mathscr{F}_{R}\left(\Gamma_{2} \mid \Lambda_{2}\right)^{1}$. Since $B \in \Gamma_{2} \backslash \Lambda_{2}, f(B) \in \overline{\Gamma_{1} \backslash \Lambda_{1}}$. Let $f(B)=D^{t}, D \in \Gamma_{1} \backslash \Lambda_{1}$, $t \in \boldsymbol{R}^{+}$. But $b A=b_{i}=f\left(a_{i}\right)=f(a) f g(A)=f(a) f(c) D^{t r}, a$ contradiction since $A \in \Lambda_{1}, D \notin \Lambda_{1}$. Thus $B \in \Lambda_{2}$ and we can assume that $g(A)=c B$ for some $c \in \mathscr{F}_{\boldsymbol{R}}\left(\Gamma_{2} \mid \Lambda_{2}\right)^{1}$. Now $b A=b_{i}=f\left(a_{i}\right)=f(a c) f(B)$. So $f(B)=d A$ for some $d \in \mathscr{F}_{\boldsymbol{R}}\left(\Gamma_{1} \mid \Lambda_{1}\right)^{1}$. Also the dual of the above argument, applied to $B$, shows that there exist $j \in\{1, \cdots, n\}, x, y \in$ $\mathscr{F}_{\boldsymbol{R}}\left(\Gamma_{2} \mid \Lambda_{2}\right)^{1}, z \in \mathscr{F}_{\boldsymbol{R}}\left(\Gamma_{1} \mid \Lambda_{1}\right)^{1}, C \in \Lambda_{1}$ such that $a_{j}=B x, f(B)=C z, g(C)=B y$. We claim that $C=A$. Suppose not. Then since $C z=f(B)=d A$, we see that $f(B)=C u A$ for some $u \in \mathscr{F}_{\boldsymbol{R}}\left(\Gamma_{1} \mid A_{1}\right)^{1}$. Then $g f(B)=$ $g(C) g(u) g(A)=B y g(u) c B$. Thus $B$ appears at least twice in $g f(B)$. An easy induction shows that $B$ appear at least $2^{i}$ times in $(g f)^{i}(B)$. In particular $l\left((g f)^{i}(B)\right) \rightarrow \infty$ as $i \rightarrow \infty$. However $a_{j}=(g f)^{i}\left(a_{j}\right)=$ $(g f)^{i}(B)(g f)^{i}(x)$ for all $i$. This contradiction shows that $A=C$. So $b_{j}=f\left(a_{j}\right)=f(B) f(x)=C z f(x)=A z f(x)$ and $b_{j}$ starts with $A$.

Next suppose $A \in \Gamma_{1} \backslash \Lambda_{1}$. There exists $i \in\{1, \cdots, n\}$ such that $b_{i}$ ends with $A$. So there exists $r \in \boldsymbol{R}^{+}, b \in \mathscr{F}_{\boldsymbol{R}}\left(\Gamma_{1} \backslash \Lambda_{1}\right)^{1}$ such that $b_{i}=b A^{r}$. Now $g(A) \in \overline{\Gamma_{2} \backslash \Lambda_{2} \text {. }}$ So there exists $k \in \boldsymbol{R}^{+}, B \in \Gamma_{2} \backslash \Lambda_{2}$ such that $g(A)=B^{k}$. Now $f(B) \in \overline{\Gamma_{1} \backslash \Lambda_{1}}$. So there exist $t \in R^{+}, C \in \Gamma_{1} \backslash \Lambda_{1}$ such that $f(B)=C^{t}$. Thus

$$
b A^{r}=b_{i}=f\left(a_{i}\right)=f g\left(b_{i}\right)=f g(b) C^{r t k} .
$$

It follows that $C=A$. There exists $j \in\{1, \cdots, n\}$ such that $a_{j}$ starts with $B$. Since $f(B)=A^{t}, b_{j}=f\left(a_{j}\right)$ starts with $A$. This completes the proof.

LEMMA 2.22. Let $\mathscr{Y}$ be a generalized word equation with solutions $\nu=\left(a_{1}, \cdots, a_{n}\right), \mu=\left(b_{1}, \cdots, b_{n}\right)$ having alphabets $\Gamma_{1}\left|\boldsymbol{\Lambda}_{1}, \Gamma_{2}\right| \Lambda_{2}$, respectively. Suppose $\nu$ satisfies (2.4) and its dual, and $f: \nu \leqq \mu$, $g: \mu \leqq \nu$. Then $g=f^{-1}$ and $f, g$ are unique. If $f_{1}, f_{2}$ are restrictions of $f$ to $\Lambda_{1}$ and $\overline{\Gamma_{1} / \Lambda_{1}}$ respectively, then $f_{1}: \Lambda_{1} \rightarrow \Lambda_{2}$ and $f_{2}: \overline{\Gamma_{1} / \Lambda_{1}} \rightarrow$ $\bar{\Gamma}_{2} / \Lambda_{2}$ are bijections.

Proof. Now $f\left(\Gamma_{1} \backslash \Lambda_{1}\right) \subseteq \overline{\Gamma_{2} \backslash \Lambda_{2}}, g\left(\Gamma_{2} \backslash \Lambda_{2}\right) \subseteq \overline{\Gamma_{1} \backslash \Lambda_{1}}$. Let $A \in \Lambda_{1}$. Then there exist $i, j \in\{1, \cdots, n\}, c, d \in \mathscr{F}_{R}\left(\Gamma_{1} \backslash \Lambda_{1}\right)^{1}$ such that $a_{i}=A C, a_{j}=d A$. So $A c=a_{i}=g f\left(a_{i}\right)=g f(A) g f(c)$. Similarly $d A=g f(d) g f(A)$. Thus $g f(A)$ starts and ends with $A$. Let $f(A)$ start with $B$, end with $C$. 
If $B \in \Gamma_{2} \backslash \Lambda_{2}$, then $(B) \in \overline{\Gamma_{1} \backslash \Lambda_{1}}$ contradicting the fact that $g f(A)$ starts with $A$. So $B \in \Lambda_{2}$. Similarly $C \in \Lambda_{2}$. So $f(A)=B x=y C$ for some $x, y \in$ $\mathscr{F}_{R}\left(\Gamma_{2} \mid \Lambda_{2}\right)^{1}$. Also $g f(A)=g(B) g(x)=g(y) g(C)$. So $g(B)$ starts with $A$ and $g(C)$ ends with $A$. So there exist $a, b \in \mathscr{F}_{R}\left(\Gamma_{1} \mid \Lambda_{1}\right)^{1}$ such that $g(B)=$ $A a, g(C)=b A$. We claim that $f(A)=B$. Otherwise there exists $z \in \mathscr{F}_{\boldsymbol{R}}\left(\Gamma_{2} \mid \Lambda_{2}\right)^{1}$ such that $f(A)=B z C$. So $g f(A)=A a z b A$. An easy induction shows that $A$ appears at least $2^{k+1}$ times in $(g f)^{k}(A)$ for all $k \in Z^{+}$. In particular $l(g f)^{k}(A) \rightarrow \infty$. But $a_{i}=(g f)^{k}\left(a^{i}\right)=(g f)^{k}(A) c$ for all $k \in Z^{+}$. This contradiction shows that $f(A)=B$. Now $g(B)=$ $A a=b A$. We claim that $A=g(B)$. Otherwise there exists $a_{1} \in$ $\mathscr{F}_{R}\left(\Gamma_{1} \mid \Lambda_{1}\right)^{1}$ such that $g(B)=A a_{1} A$. So $f g(B)=B f\left(a_{1}\right) B$. As above, this implies that $l\left((f g)^{k}(B)\right) \rightarrow \infty$ as $k \rightarrow \infty$. This contradicts the fact that $(f g)^{k}(B)$ is a segment of $(f g)^{k}\left(b_{i}\right)=b_{i}$ for all $k \in Z^{+}$. So $A=g(B)$. Thus $f\left(\Lambda_{1}\right) \subseteq \Lambda_{2}$ and $g f$ is the identity map on $\Lambda_{1}$. So $f$ is $1-1$ on $A_{1}$. Let $D \in \Lambda_{2}$. There exists $j \in\{1, \cdots, n\}$ such that $D$

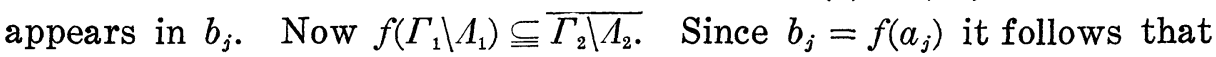
$b_{j}$ and hence $D$ lie in the semigroup generated by $\overline{\Gamma_{2} \backslash \Lambda_{2}}$ and $f\left(\Lambda_{1}\right)$. Hence $D \in f\left(\Lambda_{1}\right)$. So $f\left(\Lambda_{1}\right)=\Lambda_{2}$. Let $f_{1}$ be the restriction of $f$ to $\Lambda_{1}$. $f_{1}: \Lambda_{1} \rightarrow \Lambda_{2}$ is a bijection.

Now let $A \in \Gamma_{1} \backslash \Lambda_{1}$. There exists $i \in\{1, \cdots, n\}$ such that $a_{i}$ starts with $A$. So there exist $a \in \mathscr{F}_{\boldsymbol{R}}\left(\Gamma_{1} \mid \Lambda_{1}\right)^{1}, \boldsymbol{r} \in \boldsymbol{R}^{+}$, such that $a_{i}=A^{r} a$ and $a$ does not start with $A$. Then $f(A) \in \overline{\Gamma_{2} \backslash \Lambda_{2}}$. So there exists $B \in \Gamma_{2} \backslash \Lambda_{2}, t \in \boldsymbol{R}^{+}$such that $f(A)=B^{t}$. Now $A^{r} a=a_{i}=g f\left(a_{i}\right)=$ $g\left(B^{r t}\right) g f(a)$. So $g(B) \in \bar{A}$. Thus $g f(A) \in \bar{A}$ for all $A \in \Gamma_{1} \backslash \Lambda_{1}$. It follows that $f$ is $1-1$ on $\overline{\Gamma_{1} \backslash \Lambda_{1}}$. Since $g f\left(\Lambda_{1}\right)=\Lambda_{1}$, it also follows that in the above instance, $g f(a)$ does not start with $A$. So $g\left(B^{r t}\right)=A^{r}$. Hence $g(B)=A^{1 / t}$ and $g f$ is the identity map on $\Gamma_{1} \overline{\Lambda_{1}}$. Let $D \in \Gamma_{2} \backslash \Lambda_{2}$ Then $D$ appears in $b_{j}$ for some $j \in\{1, \cdots, n\}$. Since $f\left(a_{j}\right)=b_{j}$ it follows that $b_{j}$ and hence $D$ is an element of the semigroup generated by $f\left(\overline{\left.\Gamma_{1} \backslash \Lambda_{1}\right)}\right.$ and $\Lambda_{2}$. So $D \in f\left(\overline{\left.\Gamma_{1} \backslash \Lambda_{1}\right)}\right.$. Hence $f\left(\overline{\left.\Gamma_{1} \backslash \Lambda_{1}\right)}=\overline{\Gamma_{2} \backslash \Lambda_{2}}\right.$. So if $f_{2}$ is the restriction of $f$ to $\overline{\Gamma_{1} \backslash \Lambda_{1}}$, then $f_{2}: \overline{\Gamma_{1} \backslash \Lambda_{1}} \rightarrow \bar{\Gamma}_{2} \backslash \Lambda_{2}$ is a bijection. Also, it now follows that $f: \mathscr{F}_{R}\left(\Gamma_{1} \mid \Lambda_{1}\right) \rightarrow \mathscr{F}_{\boldsymbol{R}}\left(\Gamma_{2} \mid \Lambda_{2}\right)$ is a bijection. Since $g f$ is the identity map on $\Lambda_{1}$ and $\overline{\Gamma_{1} \backslash \Lambda_{1}}$, it is the identity map on $\mathscr{F}_{R}\left(\Gamma_{1} \mid \Lambda_{1}\right)$. So $g=f^{-1}$. If $g^{\prime}: \mu \leqq \nu$, then the above argument shows that $g^{\prime}=f^{-1}=g$. So $g$ is unique. Similarly $f$ is unique.

THEOREM 2.23. Let $\mathscr{W}$ be a generalized word equation and $\mu=\left(b_{1}, \cdots, b_{n}\right)$ a principal solution of $\mathscr{W}$ with alphabet $\Gamma \mid \Lambda$. Then for each $A \in \Gamma$, there exist $i, j \in\{1, \cdots, n\}$ such that $b_{i}$ starts with $A$ and $b_{j}$ ends with $A$.

Proof. By Theorem 2.19, there exists a principal solution $\nu$ of 
$\mathscr{W}$ satisfying (2.4) such that $f: \nu \leqq \mu$ for some $f$. By Lemma 2.21, $\nu$ satisfies the dual of (2.4). Since $\mu$ is principal, there exists $g: \mu \leqq \nu$. Thus $f$ has the structure given by Lemma 2.22. It immediately follows that $\mu$ satisfies (2.4) and its dual.

Lemma 2.22 and Theorem 2.23 imply the following.

THEOREM 2.24. Let $\mathscr{W}^{-}$be a generalized word equation with principal solutions $\nu, \mu$ having alphabets $\Gamma_{1}\left|\Lambda_{1}, \Gamma_{2}\right| \Lambda_{2}$, respectively. Suppose $f: \nu \leqq \mu$. Then $f: \mathscr{F}_{\boldsymbol{R}}\left(\Gamma_{1} \mid \Lambda_{1}\right) \cong \mathscr{F}_{\boldsymbol{R}}\left(\Gamma_{2} \mid \Lambda_{2}\right)$ is a natural isomorphism. Moreover $f$ is unique and $f^{-1}: \mu \leqq \nu$. If $f_{1}, f_{2}$ are the restrictions of $f$ to $\Lambda_{1}$ and $\overline{\Gamma_{1} \backslash \Lambda_{1}}$ respectively, then $f_{1}: \Lambda_{1} \rightarrow \Lambda_{2}$, $f_{2}: \overline{\Gamma_{1} \backslash \Lambda_{1}} \rightarrow \overline{\Gamma_{2} \backslash \Lambda_{2}}$ are bijections.

REMARK 2.25. Let $\nu, \mu$ be solutions of a generalized word equation $\mathscr{W}$ such that $\Gamma_{1}\left|\Lambda_{1}, \Gamma_{2}\right| \Lambda_{2}$ are the alphabets of $\nu, \mu$, respectively. Suppose $f: \nu \leqq \mu, g: \mu \leqq \nu$. In free semigroups (i.e., if $\Gamma_{1}=\Lambda_{1}, \Gamma_{2}=\Lambda_{2}$ ) this implies that $f, g$ are unique and $g=f^{-1}$. However, this is not true in general. In fact consider the word equation $\{X, X\}$. Let $\mu=\left(A B^{1 / 2}\right)$ with alphabet $\{A, B\} \mid\{A\}$. Then $f: \mu \leqq \mu$ where $f(A)=A B^{1 / 4}$ and $f(B)=B^{1 / 2}$. Also $I: \mu \leqq \mu$ where $I(A)=A$ and $I(B)=B$.

THEOREM 2.26. Let $\mu, \nu$ be solutions of a generalized word equation $\mathscr{W}$. Suppose $\nu$ is principal and $g: \nu \leqq \mu$. Then $g$ is unique.

Proof. By Theorem 2.19 there exists a principal solution $\delta$ of $\mathscr{Y}, f: \delta \leqq \mu$ such that $f$ is unique and $\delta \in \mathscr{S}$. Since $\delta \in \mathscr{S}$ and $\nu$ is principal, $\nu \approx \mu$. By Theorem 2.25, there exists $h$ such that $h: \delta \leqq \nu, h^{-1}: \nu \leqq \delta$. Tnen $g h: \delta \leqq \mu$. By uniqueness of $f, g h=f$. So $g=f h^{-1}$. Similarly if $g^{\prime}: \nu \leqq \mu$, then $g^{\prime}=f h^{-1}$. So $g$ is unique.

EXAMPLE 2.27. Theorem 2.26 is not true without the assumption that $\nu$ is principal. To see this let $\mathscr{W}=\{X, X\}$. Let $\nu=(A B)$, $\mu=\left(A B^{2}\right)$ in alphabet $\{A, B\}$. Let $f: \mathscr{F}(A, B) \rightarrow \mathscr{F}(A, B)$ be given by $f(A)=A B, f(B)=B$. Let $g: \mathscr{F}(A, B) \rightarrow \mathscr{F}(A, B)$ be given by $g(A)=A, g(B)=B^{2}$. Then $f: \nu \leqq \mu, g: \nu \leqq \mu$, but $f \neq g$.

DeFinItion 2.28. Let $\mathscr{W}$ be a generalized word equation and $\mu=\left(b_{1}, \cdots, b_{n}\right)$ a solution of $\mathscr{W}$. Then $\mu$ is integral if $b_{1}, \cdots, b_{n} \in$ $\mathscr{F}(\Gamma)$ for some $\Gamma . \mu$ is rational if $b_{1}, \cdots, b_{n} \in \mathscr{F}_{Q}(\Gamma)$ for some $\Gamma$.

OBSERVATION 2.29. Let $\mu, \nu$ be solutions of a generalized word 
equation $\mathscr{W}$ such that $\nu \leqq \mu$ and $\mu$ is integral. Then $\nu$ is integral.

THEOREM 2.30. Let $\mu, \nu$ be solutions of a generalized word equation $\mathscr{W}$ such that $\nu \leqq \mu, \mu$ is rational and $\nu$ is principal. Then $\nu$ is integral.

Proof. Let $\mu=\left(b_{1}, \cdots, b_{n}\right)$ with alphabet $\Gamma \mid \Lambda$. Then $b_{1}, \cdots, b_{n} \in$ $\mathscr{F}_{Q}(\Gamma)$. It follows that there exists a natural automorphism $\phi$ of $\mathscr{F}_{R}(\Gamma \mid \Lambda)$ such that $\phi$ restricted to $\mathscr{F}(\Lambda)$ is the identity map, and $\dot{\phi}\left(b_{1}\right), \cdots, \phi\left(b_{n}\right) \in \mathscr{F}(\Lambda)$. Let $a_{i}=\phi\left(b_{i}\right), i=1, \cdots, n$. Then $\mu_{1}=$ $\left(a_{1}, \cdots, a_{n}\right)$ is an integral solution of $\mathscr{W}$ and $\phi^{-1}: \mu_{1} \leqq \mu$. By Theorem 2.19, there exists a principal solution $\nu_{1}$ of $\mathscr{W}$ such that $\nu_{1} \leqq \mu$. By Observation 2.29, $\nu_{1}$ is integral. Since $\nu_{1} \leqq \mu, \nu \leqq \mu$, Corollary 2.20 implies that $\nu \approx \nu_{1}$. Again by Observation $2.29, \nu$ is integral.

CoRollary 2.31. Let $\mu$ be a rational, principal solution of a generalized word equation $\mathscr{\mathscr { W }}$. Then $\mu$ is integral.

3. Rank of a word equation. We now study the maximum of the cardinalities of the alphabets of the principal solutions of a generalized word equation. For word equations in free semigroups, this number has been studied in some detail by Lentin [2] and others.

DeFinition 3.1. Let $\mathscr{W}$ be a generalized word equation and let $\mu$ be a solution of $\mathscr{W}$ having alphabet $\Gamma \mid \Lambda$. Then rank of $\mu, R(\mu)=|\Gamma|$, the number of elements in $\Gamma$. Rank of $\mathscr{W}, R(\mathscr{W})=$ Sup. $\{R(\nu) \mid \nu$ is a principal solution of $\mathscr{W}\}$. The integral rank of $\mathscr{W}, I . R(\mathscr{W})=\operatorname{Sup} .\{R(\nu) \mid \nu$ is an integral principal solution of $\mathscr{W}\}$. We take Sup. $\varnothing$ to mean 0 .

Let $\mathscr{W}^{-}$be a generalized word equation and $\mu$ a principal solution of $\mathscr{W}$. Consider the following property of $\mu$ :

(3.2) There exists an integral principal solution $\nu$ of $\mathscr{W}$ such that $R(\nu) \geqq R(\mu)$.

Following a procedure similar to that of $\S 2$ one can show that every principal solution of a generalized word equation satisfies (3.2). The following theorem then follows,

THeOREM 3.3. Let $\mathscr{W}^{-}$be a generalized word equation. Then $R(\mathscr{W})=I \cdot R(\mathscr{W})$. 
The following theorems can also be proved in a similar manner.

THEOREM 3.4. Let $\mathscr{W}=\left\{w_{1} w_{2} ; T_{1}, \cdots, T_{s}\right\}$ be a generalized word equation with $r$ free variables. Then $R(\mathscr{W}) \leqq r+s$.

TheOREM 3.5. Let $\mathscr{W}^{-}=\left\{w_{1}, w_{2} ; T_{1}, \cdots, T_{s}\right\}$ be a generalized word equation in variables $X_{1}, \cdots, X_{n}$ such that $w_{1} \neq w_{2}$. Then $R(\mathscr{W}) \leqq n-1$.

\section{REFERENCES}

1. A. Lentin, Equations dans les monoïdes libres, Gauthier-Villars Paris, (1972).

2. - Problèmes posés en théorie des permutations par la rèsolution des equations bipermutationnelles dans les monödes libres, in: Permutations, Actes du Colloque tenu à Paris en juillet 1972 (Gauthier-Villars, Paris et Mouton, La Haye, 1974), 121-133.

3. E. S. Ljapin, Semigroups, Amer. Math. Soc. Translations 1963; translation of Polugruppy (1960).

4. M. S. Putcha, Word equations of paths, J. Algebra, (to appear).

5. - Word equations in some geometric semigroups, Pacific J. Math., 75 (1978), 243-266.

Received February 3, 1978 and in revised form September 1, 1978.

North Carolina State University

RALEIGH, NC 27650 


\section{PACIFIC JOURNAL OF MATHEMATICS}

EDITORS

DONALD BABBITT (Managing Editor)

University of California

Los Angeles, California 90024

Hugo RossI

University of Utah

Salt Lake City, UT 84112

C. C. MOORE and ANDREW OGG

University of California

Berkeley, CA 94720

\section{J. DUGUNDJI}

Department of Mathematics University of Southern California Los Angeles, California 90007

R. Finn and J. Milgram Stanford University

Stanford, California 94305

\section{ASSOCIATE EDITORS}
E. F. BECKENBACH
B. H. Neumann
F. WOLF
K. YoSHIDA

\section{SUPPORTING INSTITUTIONS}

UNIVERSITY OF BRITISH COLUMBIA

CALIFORNIA INSTITUTE OF TECHNOLOGY

UNIVERSITY OF CALIFORNIA

MONTANA STATE UNIVERSITY

UNIVERSITY OF NEVADA, RENO

NEW MEXICO STATE UNIVERSITY

OREGON STATE UNIVERSITY

UNIVERSITY OF OREGON
UNIVERSITY OF SOUTHERN CALIFORNIA

STANFORD UNIVERSITY

UNIVERSITY OF HAWAII

UNIVERSITY OF TOKYO

UNIVERSITY OF UTAH

WASHINGTON STATE UNIVERSITY

UNIVERSITY OF WASHINGTON 


\section{Pacific Journal of Mathematics}

\section{Vol. 83, No. 1 \\ March, 1979}

Richard Neal Ball, Topological lattice-ordered groups ............... 1

Stephen Berman, On the low-dimensional cohomology of some

infinite-dimensional simple Lie algebras .................. 27

R. P. Boas and Gerald Thomas Cargo, Level sets of derivatives ......... 37

James K. Deveney and John Nelson Mordeson, Splitting and modularly

perfect fields......................................

Robert Hugh Gilman and Ronald Mark Solomon, Finite groups with small

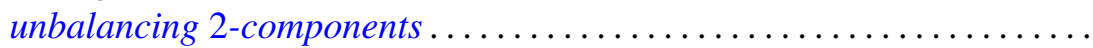

George Grätzer, Andras Hajnal and David C. Kelly, Chain conditions in free products of lattices with infinitary operations..................

Benjamin Rigler Halpern, Periodic points on tori ..................

Dean G. Hoffman and David Anthony Klarner, Sets of integers closed under

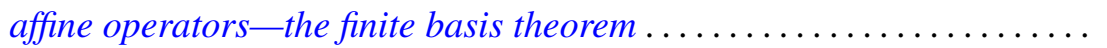

Rudolf-Eberhard Hoffmann, On the sobrification remainder ${ }^{s} X-X \ldots \ldots$

Gerald William Johnson and David Lee Skoug, Scale-invariant

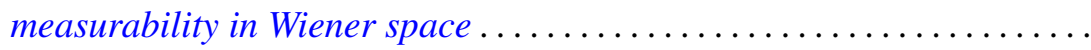

Michael Keisler, Integral representation for elements of the dual of

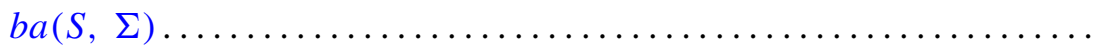

Wayne C. Bell and Michael Keisler, A characterization of the representable Lebesgue decomposition projections ................

Wadi Mahfoud, Comparison theorems for delay differential equations ...

R. Daniel Mauldin, The set of continuous nowhere differentiable functions .

Robert Wilmer Miller and Mark Lawrence Teply, The descending chain condition relative to a torsion theory...

Yoshiomi Nakagami and Colin Eric Sutherland, Takesaki's duality for regular extensions of von Neumann algebras ........ .

William Otis Nowell, Tubular neighborhoods of Hilbert cube manifolds ...

Mohan S. Putcha, Generalization of Lentin's theory of principal solutions of word equations in free semigroups to free product of copies of positive reals under addition

Amitai Regev, A primeness property for central polynomials . ...

Saburou Saitoh, The Rudin kernels on an arbitrary domain. . .

Heinrich Steinlein, Some abstract generalizations of the

Ljusternik-Schnirelmann-Borsuk covering theorem . . . 